\title{
Small Collision Systems at RHIC
}

\author{
Norbert Novitzky ${ }^{1, \star}$ \\ ${ }^{1}$ Department of Physics and Astronomy, Stony Brook University, Stony Brook, NY 11790, USA
}

\begin{abstract}
The observation of long range correlations in highly asymmetric systems, as in $p+\mathrm{Pb}$ and $d+\mathrm{Au}$ collisions, suggests a creation of a medium with collective behavior. It is still an open question if the quark-gluon plasma is formed in these collision. Hence, the RHIC collider invested time to study the small systems in different collision systems and energies. Here we discuss the recent results from the PHENIX and STAR collaborations in four different collision systems $p+\mathrm{Al}, p+\mathrm{Au}, d+\mathrm{Au}$ and ${ }^{3} \mathrm{He}+\mathrm{Au}$ at $\sqrt{s_{N N}}=200 \mathrm{GeV}$, and also for the energy scan in $d+$ Au collisions between $\sqrt{s_{N N}}=19.6-200 \mathrm{GeV}$.
\end{abstract}

\section{Introduction}

The study of non-perturbative aspects of QCD is one of the main goals of high energy nuclear collisions. In such collisions the created colored medium provides a unique way to study the QCD phase transition. Experiments at RHIC and later at LHC established the creation of such medium - called the Quark-Gluon Plasma (QGP).

The recent measurements of flow-like behavior in small-on-large collision systems at LHC [1] and RHIC energies [2] prompted a large interest in studying the possible creation of a quark-gluon droplet in these systems. The azimuthal anisotropy $\left(v_{n}\right)$ can be understood as arising from initial geometry propagated to final-state momentum correlations via interactions between medium constituents. The RHIC collaborations proposed a systematic study of the medium effects in highly asymmetric collisions. The first approach is to change the initial geometry of the collision, therefore we examined the $p+\mathrm{Au}, d+\mathrm{Au}$ and ${ }^{3} \mathrm{He}+\mathrm{Au}$ collisions at $\sqrt{s_{N N}}=200 \mathrm{GeV}$. In addition, we also investigated the dependence on collision energy and studied the $d+$ Au collisions at four different energies from 19.6 to $200 \mathrm{GeV}$.

The non-zero azimuthal anisotropy is the first observed signature of possible formation of a collective medium in small collision systems. However, the $v_{n}$ measurement alone is ambiguous as there could be other effects which would cause the measured azimuthal anisotropy. Therefore it is very important to study also the other signatures of the QGP in order to confirm the medium creation in these small systems. This proceedings focuses not only on the collective motion, but also on the jet quenching phenomena and the strangeness production in small systems.

\section{Azimuthal anisotropy measurements}

At the beginning of a heavy ion collision, the spatial distribution of the overlapping matter resembles an ellipsoid due to the incomplete overlap of the two colliding nuclei. The strong scattering in this

\footnotetext{
^e-mail: Norbert.Novitzky@ cern.ch
} 
stage converts the spatial anisotropy to a momentum anisotropy which is observable as an elliptic flow of the emitted hadrons. The azimuthal anisotropy of the spectra can be characterized in terms of Fourier coefficients. We know that the initial state fluctuations of the nuclei within the nucleus can cause a more complicated overlap area than an ellipsoid and thus result in the higher order components.

It is natural to ask how the study of collective effects in the small system will contribute to the understanding of the flow evolution as a function of the initial geometry and the multiplicity of the events. At RHIC both of the detectors collected excellent data in various collision systems to explore the initial geometry effects. In addition, RHIC provided also an energy scan in $d+$ Au collisions in order to study the onset of the observed small system collective motion as a function of the energy.

\subsection{Study of the initial geometry}

It is expected that the different collision systems will provide a different initial geometry [3]. For example, the expectation from the $d+$ Au collisions is a natural ellipticity due to the two nucleons in the deuteron, while in the ${ }^{3} \mathrm{He}+\mathrm{Au}$ collisions the initial expectation is more triangular. However, one has to be careful with the initial fluctuations of the geometrical orientation also, as the deuteron and the ${ }^{3} \mathrm{He}$ nucleus are not distributed in only the transverse plane but rather 3-dimensionally. This effect can cause various initial geometrical fluctuation which is complicating the simple picture of number of hotspots in the transverse plane. The PHENIX strategy is to utilize the high-multiplicity triggers using the beam-beam counters in order to focus on events only with large number of particles. The STAR collaboration approach is to focus on all (small and large) collision systems at fixed charge particle multiplicities.

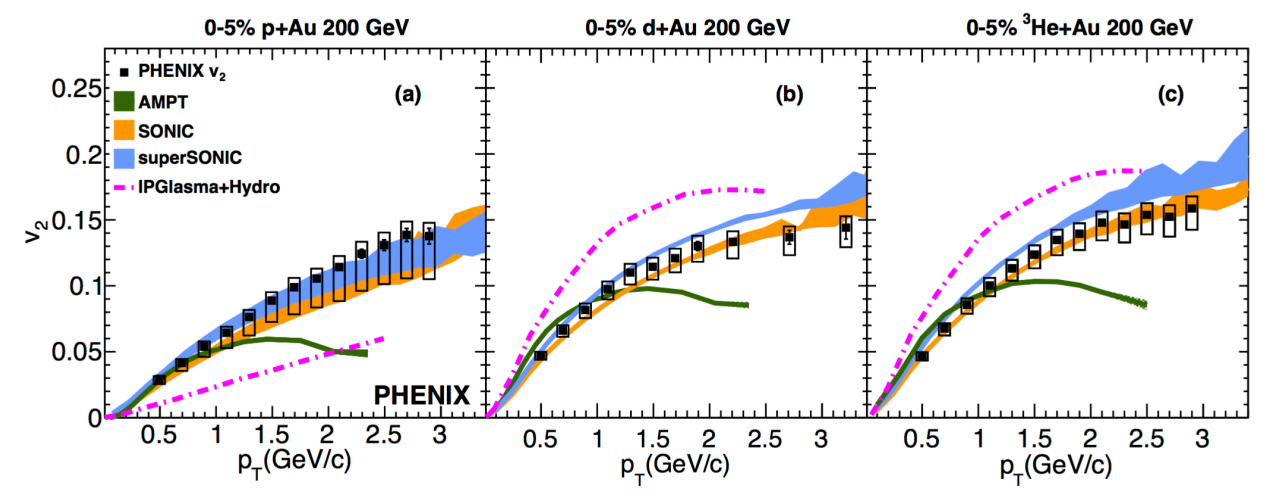

Figure 1. The measured azimuthal anisotropy by PHENIX in the $p+\mathrm{Au}, d+\mathrm{Au}$ and ${ }^{3} \mathrm{He}+\mathrm{Au}$ collisions at $\sqrt{s_{N N}}=$ $200 \mathrm{GeV}$. The data are from the new high-multiplicity trigger and restricted to $0-5 \%$ centrality.

Figure 1 shows the measured $v_{2}$ by PHENIX in $p / d /{ }^{3} \mathrm{He}+\mathrm{Au}$ collisions at $\sqrt{s_{N N}}=200 \mathrm{GeV}$ from the high-multiplicity triggered data which is then restricted to $0-5 \%$ centrality categorization. The value for $v_{2}$ is very similar in $d+\mathrm{Au}$ and ${ }^{3} \mathrm{He}+\mathrm{Au}$ collisions. The results for $p+\mathrm{Au}$ collisions suggest a smaller $v_{2}$ component in the $p+$ Au collisions at low- $p_{\mathrm{T}}$ region. The data are first compared with the AMPT [4] event generator calculation which shows good agreement with the data only in the $p_{\mathrm{T}}<$ $1 \mathrm{GeV} / c$ region. The AMPT does not contain a hydrodynamical evolution of the system, the predicted $v_{2}$ is resulting from the initial partonic and final hadronic interactions and the transition between 


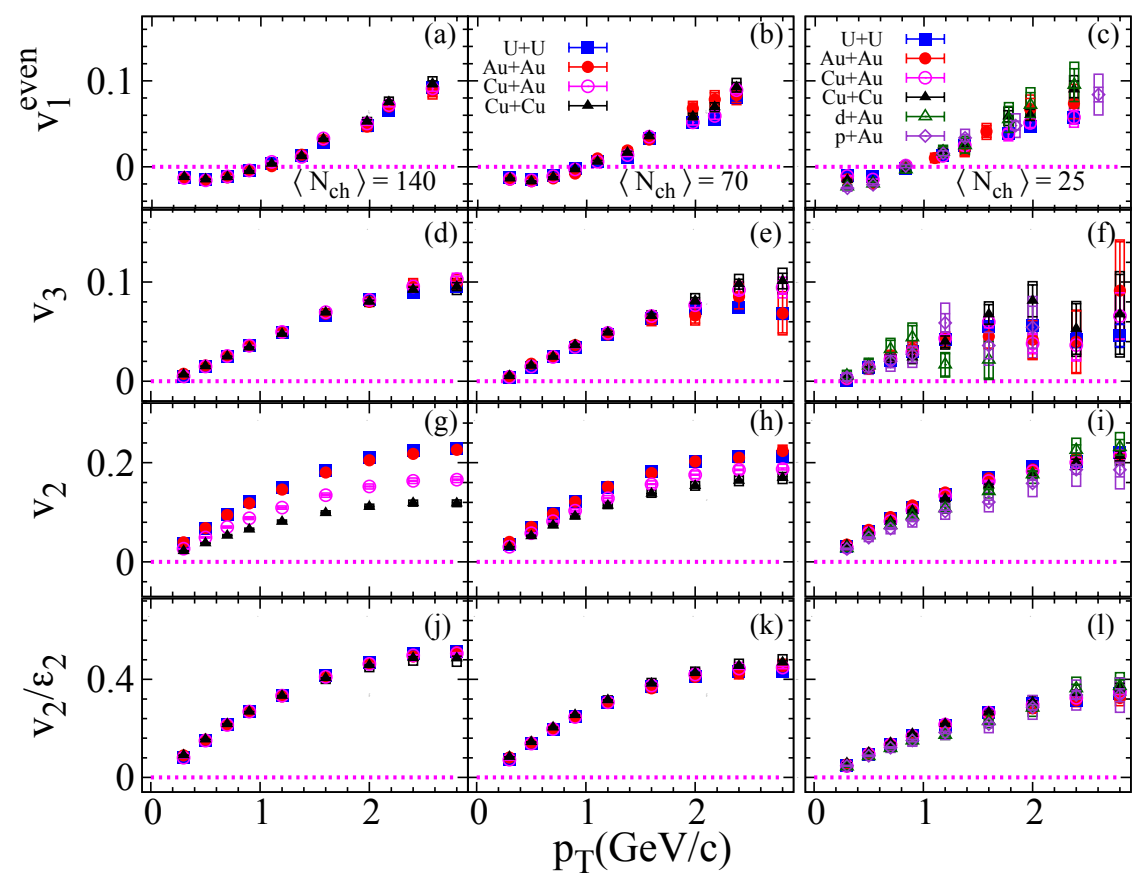

Figure 2. The measured odd (upper panels) and even (lower panels) azimuthal correlations by STAR in various collisions systems (from $p+A u$ to $\mathrm{U}+\mathrm{U}$ ) at fixed charge hadron multiplicity between $\left\langle N_{\mathrm{ch}}\right\rangle=140-25$.

these two phases of matter. The data shows excellent agreement with the hydro models SONIC and superSONIC [5], where the latter one includes the pre-equilibrium flow also. The agreement is better with the SONIC model, but the uncertainties on the data do not allow yet to invalidate the existence of the pre-equilibrium flow in these systems. The model with IPGlasma initial state and then hydrodynamical evolution [6] largely underestimates the data in $p+$ Au collisions, while overestimates the measured flow in the $d+\mathrm{Au}$ and ${ }^{3} \mathrm{He}+\mathrm{Au}$ collisions.

Figure 2 shows the measured preliminary STAR data of $v_{1}^{\text {even } \star}, v_{3}$ and $v_{2}$ measurements in various collision systems from very large $(\mathrm{U}+\mathrm{U}$ and $\mathrm{Au}+\mathrm{Au})$ to moderately large $(\mathrm{Cu}+\mathrm{Au}$ and $\mathrm{Cu}+\mathrm{Cu})$ to small systems $(d+\mathrm{Au}$ and $p+\mathrm{Au})$. The measurements are done at three fixed charged hadron multiplicities, $\left\langle N_{\mathrm{ch}}\right\rangle=140,70$ and 25 where the smallest multiplicity includes also the $p+$ Au and $d+$ Au results. The non-flow contributions are characterized as short-range and long-range effects. The short-range effect includes HBT and resonance decay contribution and they can be suppressed with a large rapidity $(\eta)$ gap. The long-range effects enter from the momentum-conservation and dijets and their contribution. In order to suppress the non-flow contributions, the results are obtained with the multi-particle correlations (sub-event cumulant method). It is important to note that these

\footnotetext{
${ }^{\star}$ In the absence of fluctuations, the directed flow $v_{1}$ develops along the direction of the impact parameter and it is an odd function of the pseudo-rapidity. However, initial-state fluctuations, acting in agreement with hydrodynamic-like expansion, gives an additional rapidity-even component $v_{1}^{\text {even }}$.
} 
studies were done only on the STAR results and thus the contributions from the non-flow effects on the data are different between the two experiments.

The $v_{1}^{\text {even }}$ and $v_{3}$ shows similar trend and magnitudes independent of the collision system only depending on the multiplicity of the event, see Figure 2. While the even harmonics, $v_{2}$, shows similar trend in different collision systems, the magnitudes depend on the collision system. However, the values can be divided by the second order eccentricities $\left(\varepsilon_{2}\right)$ of the different collision systems and then all the data scale to a single curve. The observation supports that the odd harmonics are only sensitive to fluctuations and not to the average geometries, while the second order harmonics shows significant sensitivity to the initial geometry of the collision systems [7]. In conclusion, the data suggest that at same multiplicities the initial geometry of the collision is different, although the collectivity of the system is very similar from large to small collision systems.

\subsection{Energy scan measurements}

In 2016 RHIC also delivered a beam energy scan of $d+$ Au collisions at four different collision energies from 19.6 to $200 \mathrm{GeV}$. The PHENIX measurement on these collision energies are shown in Figure 3 [8]. The data show very similar trend at all four collision energies, while the magnitude of the measured $v_{2}$ at $p_{\mathrm{T}}>1 \mathrm{GeV} / c$ is slightly increasing towards smaller collision energies. This effect is likely due to an increase in the non-flow contribution, which is not subtracted from the data.

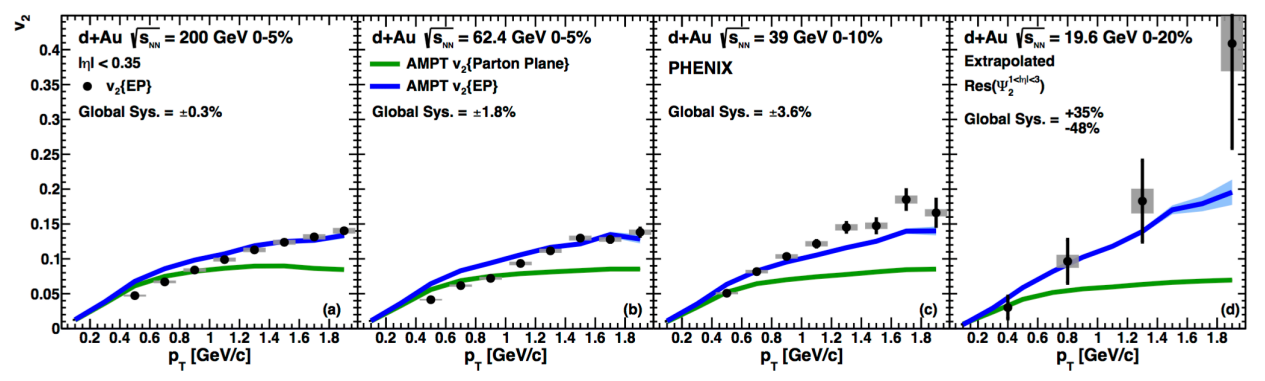

Figure 3. The measured azimuthal anisotropy by PHENIX in $d+$ Au collisions with the high-multiplicity trigger in various collision energies $\sqrt{s_{N N}}=200-19.6 \mathrm{GeV}$. The lines represent the AMPT model calculation with two different determination of the reaction plane.

The measured $v_{2}$ is compared to the AMPT calculation [4] with two different setups. The green curve shows the $v_{2}$ relative to the participant plane, so the result contains only the $v_{2}$ relative to the initial geometry. The blue curve shows the calculation of $v_{2}$ relative to the event plane. The event plane method is more consistent to the experimental extraction of the $v_{2}$ measurement, and the calculation is also in better agreement with the measured data.

\section{Searching for energy loss in small systems}

The parton propagating through the hot and dense strongly interacting medium loses part of its energy. The energy loss is characterized with the ratio of the yield in heavy ion collisions to the scaled yield in $p+p$ collisions, otherwise known as the nuclear modification factor $\left(R_{\mathrm{AA}}\right)$. The high- $p_{\mathrm{T}}$ suppression of particle production in heavy-ion collision is a clear indication of the jet energy loss [9]. The direct photons are not participating in the strong interaction with the created medium and after their creation 
they leave the medium without further interaction. The high- $p_{\mathrm{T}}$ photon measurements confirmed that there is no nuclear modification $\left(R_{\mathrm{AA}}=1\right)$, which further supports the jet energy-loss picture [10].

The energy loss is one of the key signatures in the discovery of the created plasma. Therefore, if a similar medium would be created in the small systems, one would naturally expect such nuclear modification, too. However, the picture is not so simple in the small system collisions. First, the created medium is expected to be very small, which could mean that the outgoing parton which originated from the hard scattering already left the medium before it is formed. On other hand the outgoing parton could lose energy in the cold nuclear matter. Furthermore, initial state effects can strongly affect the observed nuclear modifications. Thus it is crucial to distinguish between initial state (IS) and final state (FS) effects.

The measurement of neutral pion is a very good analysis to investigate the nuclear modification factor of the leading hadrons of jets in the small system collisions. PHENIX measured the neutral pion production in $p+\mathrm{Au}, d+\mathrm{Au}$ and ${ }^{3} \mathrm{He}+\mathrm{Au}$ minimum bias collisions at $\sqrt{s_{N N}}=200 \mathrm{GeV}$, shown in Figure 4. There is a clear enhancement observed at $p_{\mathrm{T}} \sim 5 \mathrm{GeV} / c$ in the $p+$ Au collisions, while it disappears in the $d+\mathrm{Au}$ and ${ }^{3} \mathrm{He}+\mathrm{Au}$ collisions. At high- $p_{\mathrm{T}}$ the data show some suppression $\left(R_{\mathrm{AA}}<\right.$ 1), but the uncertainties on the data are still very large.

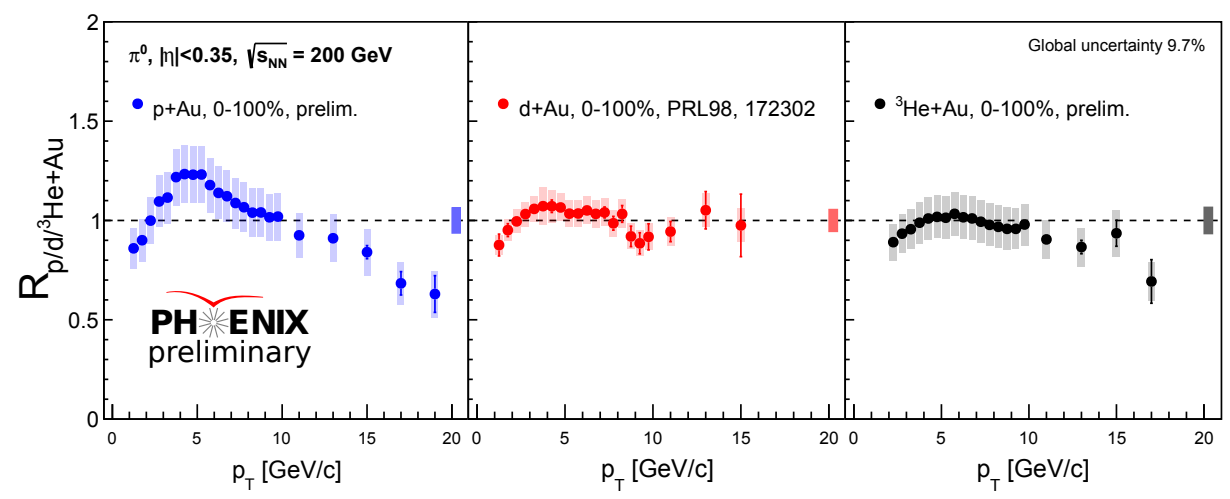

Figure 4. The nuclear modification factor of $\pi^{0}$ in $p+\mathrm{Au}, d+\mathrm{Au}$ and ${ }^{3} \mathrm{He}+\mathrm{Au}$ minimum bias collisions at $\sqrt{s_{N N}}=$ $200 \mathrm{GeV}$.

The nuclear modification factor was also measured in bins of centrality in $p+\mathrm{Au}, d+\mathrm{Au}$ and ${ }^{3} \mathrm{He}+\mathrm{Au}$ collisions. It is important to note that PHENIX uses the exact same procedure of the centrality selections using the South Beam-Beam Counter $(-3<\eta<-4)$ detector as in heavy ion collisions. Figure 5 shows the measured results in the different centrality selections in $p+\mathrm{Au}, d+\mathrm{Au}$ and ${ }^{3} \mathrm{He}+\mathrm{Au}$ collisions at $\sqrt{s_{N N}}=200 \mathrm{GeV}$. The enhancement in the mid- $p_{\mathrm{T}}$ region is more pronounced in the most central collisions while it disappears in the more peripheral collisions. The high- $p_{\mathrm{T}}$ part shows suppression at most central collisions, while in more peripheral collisions some enhancement cannot be excluded, but the data is consistent with unity within the experimental uncertainties. The nuclear modification at high- $p_{\mathrm{T}}$ is also consistent with being flat in the two most peripheral collisions.

The interpretation of the results are still ongoing as the data are being finalized. The enhancement at mid- $p_{\mathrm{T}}$ is often called the "Cronin-peak" [11] and it is traditionally attributed to the multiple scattering effect. However, the enhancement occurs at $p_{\mathrm{T}} \sim 5 \mathrm{GeV} / c$ higher than the expected contribution from multiple scattering. The high- $p_{\mathrm{T}}$ nuclear modification is very comparable within the different collisions system and the data suggest it is independent on the projectile ( $p, d$ or $\left.{ }^{3} \mathrm{He}\right)$. The data 


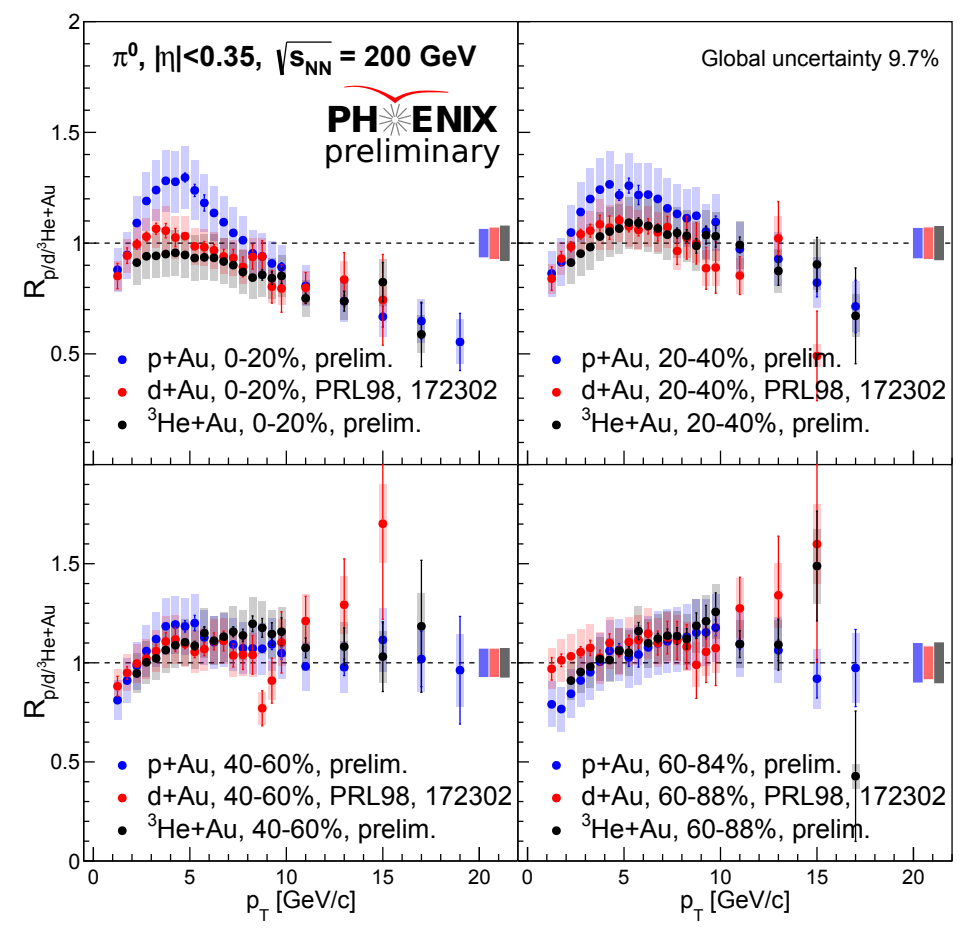

Figure 5. The nuclear modification factor of $\pi^{0}$ as a function of $p_{\mathrm{T}}$ in different centrality classes of $p+\mathrm{Au}$, $d+\mathrm{Au}$ and ${ }^{3} \mathrm{He}+\mathrm{Au}$ collisions at $\sqrt{s_{N N}}=200 \mathrm{GeV}$.

could suggest some (cold/hot) energy loss in the most central collisions [12]. The jets are suffering both IS and FS effects, while the direct photons would be sensitive only to the IS effects. In order to correctly interpret the data one needs to compare the neutral pion modification to the direct photon measurements, that are not available yet.

\section{Strangeness production in small systems}

In this section we present the recent measurements on the $\phi$ production in small system collisions from PHENIX. The $\phi$ meson is a pure strange-antistrange $(s \bar{s})$ state and its production mechanism is similar to those of $\mathrm{J} / \psi$ and $\Upsilon$. The $\phi$ meson has a small inelastic cross section with non-strange hadrons and as such represent a unique probe as they are more sensitive to the initial evolution of the system.

The forward and backward rapidity production of $\phi$ mesons was measured in $p+\mathrm{Al}, p+\mathrm{Au}$ and ${ }^{3} \mathrm{He}+$ Au minimum bias collisions at $\sqrt{s_{N N}}=200 \mathrm{GeV}$, shown in Figure 6. The data show very comparable results in all three different collision systems within the experimental uncertainties. The $\phi$ production in backward region shows enhancement, while the forward region is suppressed. The results are also very consistent with the previously measured $\phi$ production in $d+$ Au collisions. 


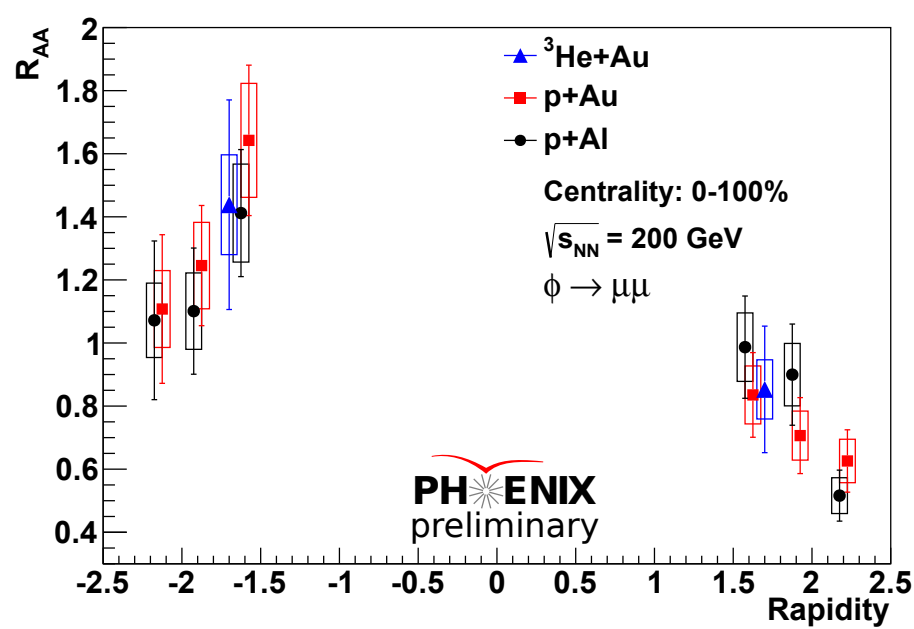

Figure 6. The nuclear modification of $\phi$ as a function of rapidity in $p+\mathrm{Al}, p+\mathrm{Au}$ and ${ }^{3} \mathrm{He}+\mathrm{Au}$ minimum bias collisions at $\sqrt{s_{N N}}=200 \mathrm{GeV}$.

\section{Summary}

This proceedings present the recent results from the RHIC collider experiment on the small collision systems. The measurement of the $v_{n}$ in the small collision systems are presented to study the initial geometrical effect as well as the energy dependence. The measurements from both PHENIX and STAR suggest strong correlation to the initial geometry of the collision system and a similarity of the observed $v_{n}$ measurements to the larger heavy-ion collision systems. Therefore the data suggest a smooth transition from $p+p$ to heavy ion collisions. The energy scan of the $d+$ Au collision shows that the long-range correlations are still present at the lowest $19.6 \mathrm{GeV}$ collision energies.

A systematic study of the neutral pion production was performed in $p / d /{ }^{3} \mathrm{He}+\mathrm{Au}$ collisions at $\sqrt{s_{N N}}=200 \mathrm{GeV}$. We observe a small enhancement in the mid- $p_{\mathrm{T}}$ region peaking around $\sim 5 \mathrm{GeV} / c$, where the data suggest an ordering between the different collision systems. The enhancement is traditionally contributed to the multiple scattering, but the peak is at much higher $p_{\mathrm{T}}$ 's than any predictions from theoretical models. At high- $p_{\mathrm{T}}$ the nuclear modification factor is very similar between the different collision systems which suggest no dependency on the size of the projectile. The $R_{\mathrm{AA}}$ values for $p_{\mathrm{T}}>10 \mathrm{GeV} / c$ show no strong $p_{\mathrm{T}}$ dependence and fall bellow unity in the most central collisions as well as in the minimum bias collisions in this $p_{\mathrm{T}}$ region. However, jet quenching is not confirmed in these collisions and the suppression could be due to multiple different mechanisms due to initial or final state effects. The direct photon measurement is needed in order to correctly interpret the results in these collision systems.

The $\phi$ meson production was measured in the forward and backward region in minimum bias $p+\mathrm{Al}, p+\mathrm{Au}$ and ${ }^{3} \mathrm{He}+\mathrm{Au}$ collisions at $\sqrt{s_{N N}}=200 \mathrm{GeV}$. The results shows small suppression in the forward region, while it is enhanced in the backward region. We also observe no significant difference between the different collision systems within the experimental uncertainties. The current results will help us study the nuclear effects in the forward and backward region in order to tune the theoretical models. 


\section{References}

[1] S. Chatrchyan et al. (CMS), Phys. Lett. B718, 795 (2013), 1210. 5482

[2] A. Adare et al. (PHENIX), Phys. Rev. Lett. 111, 212301 (2013), 1303. 1794

[3] A. Bzdak, B. Schenke, P. Tribedy, R. Venugopalan, Phys. Rev. C87, 064906 (2013), 1304 . 3403

[4] Z.W. Lin, C.M. Ko, B.A. Li, B. Zhang, S. Pal, Phys. Rev. C72, 064901 (2005), nucl-th/0411110

[5] P. Romatschke, Eur. Phys. J. C75, 305 (2015), 1502 . 04745

[6] B. Schenke, R. Venugopalan, Nucl. Phys. A931, 1039 (2014), 1407.7557

[7] B. Schenke, P. Tribedy, R. Venugopalan, Phys. Rev. C89, 064908 (2014), 1403.2232

[8] C. Aidala et al. (PHENIX) (2017), 1708.06983

[9] X.N. Wang, M. Gyulassy, Nucl. Phys. A544, 559 (1992)

[10] S. Afanasiev et al. (PHENIX), Phys. Rev. Lett. 109, 152302 (2012), 1205.5759

[11] J.W. Cronin, H.J. Frisch, M.J. Shochet, J.P. Boymond, R. Mermod, P.A. Piroue, R.L. Sumner, Phys. Rev. D11, 3105 (1975)

[12] Y.T. Chien, A. Emerman, Z.B. Kang, G. Ovanesyan, I. Vitev, Phys. Rev. D93, 74030 (2016), 1509.02936 